Discrete Comput Geom 32:345-371 (2004)

DOI: $10.1007 / \mathrm{s} 00454-004-1090-\mathrm{x}$

\title{
Convexity Properties of the Cone of Nonnegative Polynomials
}

\author{
Grigoriy Blekherman \\ Department of Mathematics, University of Michigan, \\ Ann Arbor, MI 48109-1109, USA \\ gblekher@umich.edu
}

\begin{abstract}
We study metric properties of the cone of homogeneous nonnegative multivariate polynomials and the cone of sums of powers of linear forms, and the relationship between the two cones. We compute the maximum volume ellipsoid of the natural base of the cone of nonnegative polynomials and the minimum volume ellipsoid of the natural base of the cone of powers of linear forms and compute the coefficients of symmetry of the bases. The multiplication by $\left(x_{1}^{2}+\cdots+x_{n}^{2}\right)^{m}$ induces an isometric embedding of the space of polynomials of degree $2 k$ into the space of polynomials of degree $2(k+m)$, which allows us to compare the cone of nonnegative polynomials of degree $2 k$ and the cone of sums of $2(k+m)$-powers of linear forms. We estimate the volume ratio of the bases of the two cones and the rate at which it approaches 1 as $m$ grows.
\end{abstract}

\section{Introduction and Results}

\subsection{Introduction}

Let $P_{n, d}$ denote the vector space of real homogeneous polynomials (forms) of degree $d$ in $n$ real variables. For even $d=2 k$ there are three interesting closed convex cones in $P_{n, 2 k}$ :

The cone of nonnegative polynomials, $C\left(=C_{n, 2 k}\right)$,

$$
C=\left\{f \in P_{n, 2 k} \mid f(x) \geq 0 \text { for all } x \in \mathbb{R}^{n}\right\} .
$$

The cone of sums of squares, $S q\left(=S q_{n, 2 k}\right)$,

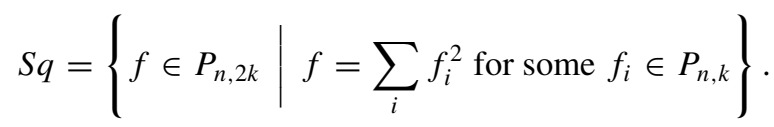


The cone of sums of $2 k$ th powers of linear forms, $L f\left(=L f_{n, 2 k}\right)$,

$$
L f=\left\{\begin{array}{l|l}
f \in P_{n, 2 k} & \left.f=\sum_{i} l_{i}^{2 k} \text { for some linear forms } l_{i} \in P_{n, 1}\right\} .
\end{array}\right.
$$

The study of algebraic properties of these cones goes back to Hilbert, who described explicitly all the cases when $C_{n, 2 k}=S q_{n, 2 k}$ [5]. Hilbert's 17th problem, solved in the affirmative by Artin and Schreier in the 1920s, asked whether every nonnegative polynomial is a sum of squares of rational functions [3]. Constructive aspects of Hilbert's problem still draws attention today [3], [9]. For a discussion of some algebraic properties of the cone of sums of powers of linear forms refer to [8].

To our knowledge, however, these cones have not been studied as general convex objects, possessing invariants based on convexity. In this paper we look at some convex properties of these cones.

Let $M\left(=M_{n, 2 k}\right)$ denote the hyperplane of all forms in $P_{n, 2 k}$ with integral 1 on the unit sphere $S^{n-1}$ :

$$
M=\left\{\begin{array}{l|l}
f \in P_{n, 2 k} & \int_{S^{n-1}} f d \sigma=1
\end{array}\right\},
$$

where $\sigma$ denotes the rotation invariant probability measure on $S^{n-1}$.

We define compact convex bodies $\bar{C}, \overline{S q}$ and $\overline{L f}$ by intersecting the respective cones with $M$ :

$$
\bar{C}=C \cap M, \quad \overline{S q}=S q \cap M \quad \text { and } \quad \overline{L f}=L f \cap M .
$$

The compact convex bodies $\bar{C}, \overline{S q}$ and $\overline{L f}$ are natural bases of the respective cones and they have full dimension in $M$. Their naturality becomes apparent if we consider the following action of the special orthogonal group $S O(n)$ on $P_{n, d}$ :

$$
A \in S O(n) \text { sends } f(x) \in P_{n, d} \quad \text { to } \quad A f=f\left(A^{-1} x\right) .
$$

All three cones $C, S q$ and $L f$ are fixed by the action of $S O(n)$, and $M$ is the only hyperplane in $P_{n, 2 k}$ fixed by this action. Therefore $\bar{C}, \overline{S q}$ and $\overline{L f}$ are also fixed by the action of $S O(n)$, and they are the only hyperplane sections of their respective cones with this property. This action of $S O(n)$ naturally gives a homomorphism

$$
\varphi_{n, d}: S O(n) \rightarrow G L\left(P_{n, d}\right),
$$

and therefore we have a representation of $S O(n)$ on $P_{n, d}$. There is a natural inner product on $P_{n, d}$ :

$$
\langle f, g\rangle=\int_{S^{n-1}} f g d \sigma .
$$

The metric induced by this inner product makes $\varphi_{n, d}$ an orthogonal representation, since the inner product is invariant under the action of $S O(n)$.

Let $K(2 m)$ be the cone in $P_{n, 2 k}$ of forms whose restrictions to the sphere are linear combinations of $2 m$ th powers of linear forms on $S^{n-1}$. Equivalently, $K(2 m)$ is the cone 
of forms in $P_{n, 2 k}$ that multiplied by $\left(x_{1}^{2}+\cdots+x_{n}^{2}\right)^{m-k}$ become sums of powers of linear forms:

$$
K(2 m)=\left\{f \in P_{n, 2 k} \mid\left(x_{1}^{2}+\cdots+x_{n}^{2}\right)^{m-k} f \in L f_{n, 2 m}\right\} .
$$

We define $\bar{K}(2 m)$ by intersecting $K$ with the hyperplane of forms of integral 1 on $S^{n-1}$.

From general convexity we know that every compact convex body $K$ contains a unique ellipsoid of maximum volume, known as John's ellipsoid of $K$. Also, $K$ is contained in a unique ellipsoid of minimum volume, known as the Loewner ellipsoid of $K$ [1].

A crude, yet interesting, measure of symmetry of $K$ is its coefficient of symmetry about a point $v$ in the interior of $K$. The coefficient of symmetry of $K$ about $v$ is defined as the largest $\alpha \in \mathbb{R}$ such that

$$
-\alpha(K-v) \text { is contained in } K-v .
$$

We will compute coefficients of symmetry of $\bar{C}$ and $\overline{L f}$ with respect to $v=$ $\left(x_{1}^{2}+\cdots+x_{n}^{2}\right)^{k}$.

\subsection{Convexity Results}

We prove the following properties:

1. Let $\alpha=\operatorname{dim} P_{n, 2 k}-1$. Then

$$
\left(\frac{\operatorname{vol} \bar{K}(2 m)}{\operatorname{vol} \bar{C}}\right)^{1 / \alpha} \geq \frac{m ! \Gamma((2 m+n) / 2)}{(m-k) ! \Gamma((2 m+2 k+n) / 2)} .
$$

It follows that if for an $\varepsilon>0$ we let $m=\left(2 k^{2}+k n\right) / \varepsilon$, then

$$
\left(\frac{\operatorname{vol} \bar{K}(2 m)}{\operatorname{vol} \bar{C}}\right)^{1 / \alpha} \geq 1-\varepsilon
$$

(see Theorem 7.8). Thus the volume ratio approaches 1 as $m$ goes to infinity. Therefore all strictly positive polynomials lie in some $K(2 m)$ (see [9]).

2. We show that, in the above metric, John's ellipsoid of $\bar{C}_{n, 2 k}$ is a ball centered at $\left(x_{1}^{2}+\cdots+x_{n}^{2}\right)^{k}$ of radius

$$
\frac{1}{\sqrt{\left(\begin{array}{c}
n+2 k-1 \\
2 k
\end{array}\right)-1}}
$$

(see Theorem 5.3).

3. We explicitly compute the Loewner ellipsoid of $\overline{L f}_{n, 2 k}$ (see Theorem 7.6).

4. We calculate the coefficient of symmetry of $\bar{C}_{n, 2 k}$ and $\overline{L f}_{n, 2 k}$ with respect to $\left(x_{1}^{2}+\cdots+x_{n}^{2}\right)^{k}$, which in both cases turns out to be

$$
\frac{1}{\left(\begin{array}{c}
n+k-1 \\
k
\end{array}\right)-1}
$$

(see Theorems 6.5 and 7.6). 
5. Combining properties 2 and 3 we show that $\overline{L f}_{n, 2 k}$ contains a ball of radius

$$
\frac{k ! \Gamma(k+n / 2)}{\Gamma(2 k+n / 2) \sqrt{\left(\begin{array}{c}
n+k-1 \\
k
\end{array}\right)-1}},
$$

centered at $\left(x_{1}^{2}+\cdots+x_{n}^{2}\right)^{k}$ (see Corollary 7.7).

6. A crucial tool for the above calculations is computation of the Loewner ellipsoid of a convex hull of the orbit of an arbitrary point under a continuous, multiplicity free group action of a compact group. We apply this to the case of $S O(n)$ (see Theorem 3.1). The case of continuous representation with no restriction on mulitiplicities will be discussed elsewhere.

Note that the invariants computed in properties 2-4 are independent of the Euclidean structure on $P_{n, d}$, e.g. the maximal volume ellipsoid is unique and is the same regardless of the choice of an inner product, although it will not always be a ball.

In many cases we reduce our calculations to polynomials symmetric with respect to an axis. These are the polynomials fixed by $J(n, v)$, where $J(n, v)$ is the subgroup of $S O(n)$ consisting of orthogonal transformations that fix a particular vector $v \in \mathbb{R}^{n}$ :

$$
J(n, v)=\left\{A \in S O(n) \mid A v=v \text { for some fixed } v \in \mathbb{R}^{n}\right\}
$$

We show that every nonnegative polynomial symmetric with respect to an axis is a sum of squares, which proves to be quite useful, and we think interesting in itself.

\subsection{Integral Inequalities}

A byproduct of our work is a number of integral inequalities for homogeneous polynomials on $S^{n-1}$. We use the usual notation for $L^{p}$ and $L^{\infty}$ norms:

$$
\|f\|_{p}=\left(\int_{S^{n-1}}|f|^{p} d \sigma\right)^{1 / p} \quad \text { and } \quad\|f\|_{\infty}=\max _{x \in S^{n-1}}|f(x)| .
$$

We list some of our results roughly in order of appearance in the paper:

1. For nonnegative $f \in P_{n, 2 k}$,

$$
\|f\|_{\infty} \leq\left(\begin{array}{c}
n+k-1 \\
k
\end{array}\right)\|f\|_{1}
$$

(see Theorem 6.3).

2. Equivalently to 1 , let $M_{f}$ denote the maximum of $f$ on $S^{n-1}$ and $m_{f}$ denote the minimum of $f$ on $S^{n-1}$. Also let $\alpha=1 /\left(\begin{array}{c}n+k-1 \\ k\end{array}\right)$. Then for all $f \in P_{n, 2 k}$,

$$
\alpha M_{f}+(1-\alpha) m_{f} \leq \int_{S^{n-1}} f d \sigma \leq(1-\alpha) M_{f}+\alpha m_{f}
$$

(see Corollary 6.6). 
3. For $f$ as in 1 ,

$$
\|f\|_{2} \leq \sqrt{\left(\begin{array}{c}
n+k-1 \\
k
\end{array}\right)}\|f\|_{1}
$$

(see Corollary 6.7).

4. From 1 we easily derive that for $f \in P_{n, k}$,

$$
\|f\|_{\infty} \leq\left(\begin{array}{c}
n+k l-1 \\
k l
\end{array}\right)^{1 / 2 l}\|f\|_{2 l}
$$

for all positive integers $l$ (see Corollary 6.4).

Estimates 1 and 2 above are sharp and we also provide all extreme polynomials for them. For a different proof of 4 by Barvinok and a discussion of applications see [2]. Sogge in [11], and Duoandikoetxea in [4] derive some related interesting inequalities.

The rest of the article is structured as follows: Section 2 contains the known results necessary for the rest of the paper. In Section 3 we compute the Loewner ellipsoid of an orbit of a point under the action of a compact group. In Section 4 we prove some results about polarity in the space of forms with respect to our inner product $\langle$,$\rangle . In Section 5$ we compute John's ellipsoid for the cone of nonnegative polynomials. In Section 6 we compute the coefficient of symmetry of the cone of nonnegative polynomials. Section 7 is devoted to the cone of sums of powers of linear forms. We derive the equation of its Loewner ellipsoid and compute its coefficient of symmetry, and we show the volume ratio result.

\section{Preliminaries}

\subsection{Representation of $S O(n)$ in $P_{n, d}$}

There is a natural action of $S O(n)$ on $P_{n, d}$ which sends $f(x)$ to $f\left(A^{-1} x\right)$ for $A \in S O(n)$. We denote the action of $A \in S O(n)$ on $f$ by $A f$. Note that this leads naturally to a representation $\varphi_{n, d}$ of $S O(n)$. We introduce an inner product on $P_{n, d}$ :

$$
\langle f, g\rangle=\int_{S^{n-1}} f(x) g(x) d \sigma,
$$

where $\sigma$ denotes the rotation invariant probability measure on $S^{n-1}$. Under our inner product the norm of $f$ coincides with the usual $L^{2}$ norm and we will often use $\|f\|$ instead of $\|f\|_{2}$. The metric induced by the inner product makes $\varphi_{n, d}$ into an orthogonal representation as

$$
\langle A f, A g\rangle=\int_{S^{n-1}} f\left(A^{-1} x\right) g\left(A^{-1} x\right) d \sigma=\int_{S^{n-1}} f(x) g(x) d \sigma=\langle f, g\rangle,
$$

by rotational invariance of $\sigma$.

We use $\Delta$ to denote the Laplace differential operator:

$$
\Delta=\frac{\partial^{2}}{\partial x_{1}^{2}}+\cdots+\frac{\partial^{2}}{\partial x_{n}^{2}} .
$$


Definition. If $f \in P_{n, d}$ and

$$
\Delta f=0,
$$

then $f$ is called a homogeneous harmonic.

The restriction of a homogeneous harmonic to the sphere $S^{n-1}$ is called a spherical harmonic. By linearity of $\Delta$, homogeneous harmonics form a vector subspace of $P_{n, d}$, which we denote by $H_{n, d}$ :

$$
H_{n, d}=\left\{f \in P_{n, d} \mid \Delta f=0\right\} .
$$

Let

$$
r(x)=\left(x_{1}^{2}+\cdots+x_{n}^{2}\right)^{1 / 2} .
$$

The inclusion $i: H_{n, d-2 l} \rightarrow P_{n, d}$ given by

$$
i(f)=r^{2 l} f
$$

is an isometry since $i(f)=r^{2 l} f$ is the same function as $f$ on the sphere $S^{n-1}$. We denote the image subspace of $P_{n, d}$ by $H_{n, d-2 l}^{*}$ :

$$
H_{n, d-2 l}^{*}=\left\{f \in P_{n, d} \mid f=r^{2 l} g \text { for some } g \in H_{n, d-2 l}\right\} .
$$

We need some facts about the representations $\varphi_{n, d}$, see [7] and [13].

Theorem 2.1. $\quad H_{n, d}$ is an irreducible $S O(n)$-module, and, therefore, $H_{n, d}^{*}$ is an irreducible submodule of $P_{n, d}$. Furthermore, $P_{n, d}$ splits into irreducible submodules as follows:

$$
P_{n, d}=\bigoplus_{i=0}^{\lfloor d / 2\rfloor} r^{2 i} H_{n, d-2 i}=\bigoplus_{i=0}^{\lfloor d / 2\rfloor} H_{n, d-2 i}^{*} .
$$

Let $D(n, d)$ be the dimension of $P(n, d)$ and let $N(n, d)$ be the dimension of $H_{n, d}$. Then

$$
D(n, d)=\left(\begin{array}{c}
n+d-1 \\
d
\end{array}\right) \quad \text { and } \quad N(n, d)=\frac{(2 d+n-2)(d+n-3) !}{d !(n-2) !} .
$$

Remark 2.2. The restriction of $f \in P_{n, d}$ to the sphere $S^{n-1}$ can be uniquely written as a sum of spherical harmonics of degrees having the same parity as $d$.

Definition. Let $J(n, v)$ denote the subgroup of $S O(n)$ that keeps a particular $v \in S^{n-1}$ fixed:

$$
J(n, v)=\{A \in S O(n) \mid A v=v\} .
$$

We denote the standard basis of $\mathbb{R}^{n}$ by $e_{1} \cdots e_{n}$. We use the following theorem on restricting $\varphi_{n, d}$ to $J(n, v)$ : 
Theorem 2.3. There exists a unique polynomial $L_{n, d}^{v}(x)$ with the following properties:

1. $L_{n, d}^{v}(x) \in H_{n, d}$,

2. $L_{n, d}^{v}(A x)=L_{n, d}^{v}(x)$ for all $A \in J(n, v)$,

3. $L_{n, d}^{v}(v)=1$.

We call $L_{n, d}^{v}(x)$ the Legendre harmonic with axis $v$ (also called the axial and zonal polynomial).

We denote $L_{n, d}^{e_{n}}$ simply by $L_{n, d}$. We now state some facts about Legendre harmonics that will be used later:

\section{Theorem 2.4.}

1. The norm of the Legendre harmonic is given by

$$
\left\|L_{n, d}^{v}\right\|^{2}=\int_{S^{n-1}}\left(L_{n, d}^{v}\right)^{2} d \sigma=\frac{1}{N(n, d)} .
$$

2.

$$
\left\|L_{n, d}^{v}\right\|_{\infty}=1 .
$$

The maximum absolute value of $L_{n, d}^{v}$ is achieved only at $v,-v$ and

$$
L_{n, 2 k}^{v}(v)=L_{n, 2 k}^{v}(-v)=1 \quad \text { while } \quad L_{n, 2 k-1}^{v}(v)=-L_{n, 2 k-1}^{v}(-v)=1 .
$$

Since $L_{n, d}$ is fixed by $J\left(n, e_{n}\right)$, by applying rotations of $S^{n-1}$ fixing $e_{n}$, we see that $L_{n, d}$ is constant on slices of the sphere with hyperplanes $T_{a}$ perpendicular to $e_{n}$ :

$$
T_{a}=\left\{\zeta \in \mathbb{R}^{n} \mid\left\langle\zeta, e_{n}\right\rangle=a\right\}, \quad-1 \leq a \leq 1 .
$$

Hence the Legendre harmonics $L_{n, d}$ restricted to the sphere $S^{n-1}$ are functions of essentially only one variable, namely, the last coordinate. Therefore we can define a polynomial in $t$, which we denote $Q_{n, d}(t)$, such that

$$
L_{n, d}(\xi)=Q_{n, d}\left(\left\langle\xi, e_{n}\right\rangle\right) \quad \text { for all } \quad \xi \in S^{n-1} .
$$

The family of polynomials $Q_{n, d}(t)$ are known as the Legendre polynomials and are special cases of ultraspherical (or Gegenbauer) polynomials. For many identities satisfied by these polynomials see [12] and [13].

\subsection{Loewner and John Ellipsoids}

Let $K$ be a convex body in a finite-dimensional real vector space $V$. There exists a unique ellipsoid of maximal volume contained in $K$, known as John's ellipsoid of $K$; we denote it by $D_{K}$. Moreover, there is a criterion for determining whether a given ellipsoid $E$ contained in $K$ is John's ellipsoid of $K$ based solely on the points in the intersection of boundaries $\partial E \cap \partial K$. 
Recall that a nonsingular linear transformation does not affect ratios of volumes. Therefore, after translating the center of $D_{K}$ to the origin and then applying a linear transformation $A \in G L(V)$, we know that John's ellipsoid of $A(K)$ is the unit ball $B^{n}$. Therefore we assume that John's ellipsoid of $K$ is a ball and we state the theorem for this case:

Theorem 2.5. Each convex body $K$ contains a unique ellipsoid of maximal volume. This ellipsoid is $B^{n}$ if and only if the following conditions are satisfied: $B^{n} \subset K$ and (for some $m$ ) there exist unit vectors $\left(u_{i}\right)_{1}^{m}$ in the boundary $\partial K$ of $K$ and positive numbers $\left(c_{i}\right)_{1}^{m}$ satisfying

$$
\sum c_{i} u_{i}=0
$$

and

$$
\sum c_{i}\left\langle x, u_{i}\right\rangle^{2}=\|x\|^{2} \quad \text { for all } \quad x \in \mathbb{R}^{n} .
$$

For the proof and discussion see [1].

There also exists a unique ellipsoid of minimal volume containing $K$, known as the Loewner ellipsoid of $K$; we denote it by $L_{K}$. It was shown by John in [6] that if $B^{n}$ contains $K$, then the same condition on points in the intersection of boundaries is necessary and sufficient for a unit ball $B^{n}$ to be the Loewner ellipsoid of $K$.

Definition. For a convex body $K$ in $V$ we use $K^{\circ}$ to denote the polar of $K$,

$$
K^{\circ}=\left\{x \in \mathbb{R}^{n} \mid\langle x, y\rangle \leq 1 \text { for all } y \in K\right\} .
$$

The following proposition relating John and Loewner ellipsoids of polar bodies will be useful later.

Proposition 2.6. Let $L_{K}$ be the Loewner ellipsoid of $K$ and suppose that the center of $L_{K}$ is the origin. Then John's ellipsoid of $K^{\circ}$ is $L_{K}^{\circ}$.

Now we assume that the center of the Loewner ellipsoid of $K$ is the origin and let $\alpha$ be the coefficient of symmetry of $K$ with respect to zero, i.e. let $\alpha$ be the largest positive real number such that

$$
-\alpha K \subseteq K
$$

Proposition 2.7. Let $K$ be a convex body and let $\alpha$ be the coefficient of symmetry of $K$ with respect to the center of the Loewner ellipsoid $L_{K}$. Then

$$
\sqrt{\frac{\alpha}{\operatorname{dim} V}} L_{K} \subseteq K \subseteq L_{K} .
$$

Similarly, if $\beta$ is the coefficient of symmetry of $K$ with respect to the center of John's ellipsoid $D_{K}$, then

$$
D_{K} \subseteq K \subseteq \sqrt{\frac{\operatorname{dim} V}{\beta}} D_{K}
$$


Proof. We show the proposition only for the case of the Loewner ellipsoid. The other case follows by polarity. Without loss of generality we may assume that $L_{K}$ is a unit ball centered at the origin. John in [6] has also shown the following:

For a unit vector $v \in \mathbb{R}^{n}$ let $d(v)$ be the distance from the origin of the supporting hyperplane of $K$ in the direction of $v$ :

$$
d(v)=\max _{x \in K}\langle x, v\rangle .
$$

Then

$$
d(v) d(-v) \geq \frac{1}{\operatorname{dim} V}
$$

Now let $w \in K$ be such that

$$
\langle v, w\rangle=d(v) .
$$

Since the coefficient of symmetry of $K$ is $\alpha$, it follows that

$$
-\alpha w \in K \quad \text { and } \quad\langle-\alpha w,-v\rangle=\alpha d(v) .
$$

Therefore we see that

$$
\alpha d(v) \leq d(-v)
$$

and thus

$$
\frac{d^{2}(-v)}{\alpha} \geq d(v) d(-v) \geq \frac{1}{\operatorname{dim} V} .
$$

Hence it follows that for all $v \in \mathbb{R}^{n}$,

$$
d(v) \geq \sqrt{\frac{\alpha}{\operatorname{dim} V}},
$$

and therefore $K$ contains a ball of radius $\sqrt{\alpha / \operatorname{dim} V}$.

\section{Loewner Ellipsoid of an Orbit}

Let $V$ be a finite-dimensional real vector space. Let $G$ be a compact topological group and let $\varphi: G \rightarrow G L(V)$ be a continuous representation of $G$. There exists a $G$-invariant probability measure $\mu$ on $G$, called the Haar measure. From the existence of the Haar measure it easily follows that there exists a $G$-invariant scalar product $\langle$,$\rangle that makes \varphi$ into an orthogonal representation [10].

We will use the shorthand notation of $g(v)$ to denote the action of $\varphi(g)$ on a vector $v \in V$. Let $v \in V$ and let $O_{v}$ be the orbit of $v$,

$$
O_{v}=\{g(v) \mid g \in G\} .
$$

Let $W$ denote the affine span of $O_{v}$,

$$
W=\left\{\sum \lambda_{i} g_{i}(v) \mid g_{i} \in G \text { and } \lambda_{i} \in \mathbb{R} \text { such that } \sum \lambda_{i}=1\right\},
$$


and let $K_{v}$ be the convex hull of $O_{v}$,

$$
K_{v}=\left\{\sum \lambda_{i} g_{i}(v) \mid g_{i} \in G, \lambda_{i} \in \mathbb{R} \text { such that } \sum \lambda_{i}=1 \text { and } \lambda_{i} \geq 0\right\} .
$$

Since $G$ is compact, it follows that $O_{v}$ is compact. Therefore $K_{v}$ is a full-dimensional compact convex set in $W$.

Let $\bar{v}$ denote the projection of $v$ into the isotypic component of $V$ corresponding to the trivial representation:

$$
\bar{v}=\int_{G} g(v) d \mu .
$$

Since $\mu$ is normalized to 1 , it follows that $\bar{v} \in W$. Now consider the linear subspace $\bar{W}$ which is obtained by subtracting $\bar{v}$ from $W$ :

$$
\bar{W}=\{w-\bar{v} \mid w \in W\} .
$$

Notice that $\bar{W}$ is a $G$-module. Since

$$
g(v-\bar{v})=g(v)-g(\bar{v})=g(v)-\bar{v},
$$

it follows that $\bar{W}$ is an affine span of $O_{v-\bar{v}}$, and $K_{v-\bar{v}}$ is $K_{v}-\bar{v}$. Thus instead of $K_{v}$ we can consider $K_{v-\bar{v}}$, inside $\bar{W}$.

Therefore we have reduced our problem to computing the Loewner ellipsoid for a point whose orbit spans the entire space affinely. Let $v \in V$ and, without loss of generality, assume that

$$
V=\operatorname{Aff}\left\{O_{v}\right\} .
$$

In this case $G$ does not fix any vector in $V$ except for the origin. For suppose not, and let $w \in V$ be fixed by $G$. Then

$$
\langle g(v), w\rangle=\left\langle g^{-1} g(v), g^{-1} w\right\rangle=\langle v, w\rangle \quad \text { for all } g \in G .
$$

Therefore

$$
\langle x, w\rangle=\langle v, w\rangle=\mathrm{const} \quad \text { for all } \quad x \in \operatorname{Aff}\left(O_{v}\right)=V .
$$

Thus $w=0$.

We now assume that the representation $\varphi$ is multiplicity free, i.e. isomorphic irreducible submodules occur with multiplicity 1 in the decomposition on $V$; the case of an arbitrary representation will be discussed elsewhere. We let

$$
V=\bigoplus_{i=1}^{k} V_{i}
$$

be the unique orthogonal decomposition of $V$ into irreducible submodules, and let $D_{i}$ be the dimension of $V_{i}$, with $D$ denoting the dimension of $V$. For $x \in V$ we use $l_{i}(x)$ to denote orthogonal projection of $x$ into $V_{i}$. Now we prove the main theorem of this section: 
Theorem 3.1. The Loewner ellipsoid $L$ of $K_{v}$ is given by the inequality

$$
\sum_{i=1}^{k} \frac{D_{i}}{\left\|l_{i}(v)\right\|^{2}}\left\|l_{i}(x)\right\|^{2} \leq D
$$

Proof. Since $K_{v}$ is the convex hull of the orbit of $v$ it follows that $K_{v}$ is fixed by the action of $G$. By the uniqueness of the Loewner ellipsoid, it follows that $L$ is also fixed under the action of $G$.

Now let $E$ be an ellipsoid in $V$ such that $E$ is fixed under the action of $G$ and $v \in E$. From invariance of $E$ under $G$ it follows that

$$
O_{v} \subseteq E,
$$

and hence

$$
K_{v} \subseteq E .
$$

We will minimize the volume of $E$, and then we will obtain the Loewner ellipsoid $L$.

Let $w$ be the center of $E$. Since $G$ fixes $E$, it follows that $G$ also fixes $w$. However, the only vector fixed by $G$ in $V$ is the origin, and thus $w=0$. Also, from the invariance of $E$ under the action of $G$, it follows that the defining inequality of $E$ must have the form

$$
\sum_{i=1}^{k} \lambda_{i}\left\|l_{i}(x)\right\|^{2} \leq 1 \quad \text { for some } \quad \lambda_{i} \in \mathbb{R} \quad \text { with } \quad \lambda_{i} \geq 0 .
$$

To minimize the volume of $E$ we may assume that $v \in \partial E$, or, in other words,

$$
\sum_{i=1}^{k} \lambda_{i}\left\|l_{i}(v)\right\|^{2}=1
$$

Also,

$$
\operatorname{vol}^{2}(E)=\operatorname{vol}^{2}\left(B^{D}\right) \prod_{i=1}^{k} \lambda_{i}^{-D_{i}},
$$

where $B^{D}$ denotes the $D$-dimensional unit ball. Thus we need to minimize

$$
\prod_{i=1}^{k} \lambda_{i}^{-D_{i}}
$$

subject to

$$
\sum_{i=1}^{k} \lambda_{i}\left\|l_{i}(v)\right\|^{2}=1
$$

We apply the method of Lagrange multipliers and it follows that

$$
c D_{i} \frac{\operatorname{vol}^{2}(E)}{\lambda_{i}}=\left\|l_{i}(v)\right\|^{2} \quad \text { for some } \quad c \in \mathbb{R}, \quad \text { and for all } \quad 1 \leq i \leq k .
$$


Therefore

$$
\lambda_{i}\left\|l_{i}(v)\right\|^{2}=c D_{i} \operatorname{vol}^{2}(E) .
$$

We substitute this into (1) and it follows that

$$
c=\frac{1}{\operatorname{vol}^{2}(E) \sum_{i=1}^{k} D_{i}}=\frac{1}{\operatorname{vol}^{2}(E) D} .
$$

This we substitute into (2) and we see that

$$
\lambda_{i}=\frac{D_{i}}{\left\|l_{i}(v)\right\|^{2} D}
$$

Now the theorem follows.

\section{Duality}

In this section we explicitly compute the dual cone of cone $C$ of nonnegative polynomials and describe some of its properties.

Definition. For $f \in P_{n, d}$ let $l_{d-2 i}(f)$ denote the projection of $f$ into $H_{n, d-2 i}^{*}$.

Theorem 4.1. For $v \in S^{n-1}$ let $p_{v} \in P_{n, d}$ be as follows:

$$
p_{v}=\sum_{i=0}^{\lfloor d / 2\rfloor} N(n, d-2 i) r^{2 i} L_{n, d-2 i}^{v}
$$

Then for all $f \in P_{n, d}$,

$$
\left\langle p_{v}, f\right\rangle=f(v)
$$

Proof. We observe that

$$
\left\langle p_{v}, f\right\rangle=\left\langle\sum_{i=0}^{k} l_{d-2 i}\left(p_{v}\right) \sum_{i=0}^{k} l_{d-2 i}(f)\right\rangle=\sum_{i=0}^{k}\left\langle N(n, d-2 i) r^{2 i} L_{n, d-2 i}^{v}, l_{d-2 i}(f)\right\rangle .
$$

Therefore it would suffice to show that for all $f \in H_{n, d-2 i}^{*}$,

$$
\left\langle N(n, d-2 i) r^{2 i} L_{n, d-2 i}^{v}, f\right\rangle=f(v) .
$$

Let $T_{v}$ denote the hyperplane of all polynomials in $H_{n, d-2 i}^{*}$ with zero at $v$,

$$
T_{v}=\left\{f \in H_{n, d-2 i}^{*} \mid f(v)=0\right\} .
$$

Since $T_{v}$ is a hyperplane, its orthogonal complement in $H_{n, d-2 i}^{*}$ is a line. Let $g \in T_{v}^{\perp}$. We observe that $T_{v}$ is fixed by the action of $J(n, v)$. Therefore $T_{v}^{\perp}$ is also fixed by $J(n, v)$, 
and from Theorem 2.3 it follows that $g=c r^{2 i} L_{n, d-2 i}^{v}$ for some constant $c \in \mathbb{R}$. Let $g=N(n, d-2 i) r^{2 i} L_{n, d-2 i}^{v}$. Since $g \in T_{v}^{\perp}$ it follows that for all $f \in H_{n, d-2 i}^{*}$,

$$
\langle f, g\rangle=c f(v) \quad \text { for some constant } \quad c \in \mathbb{R} \text {. }
$$

To compute $c$, we use $f=r^{2 i} L_{n, d-2 i}^{v}$ and observe that

$$
\left\langle r^{2 i} L_{n, d-2 i}^{v}, g\right\rangle=N(n, d-2 i)\left\langle L_{n, d-2 i}^{v}, L_{n, d-2 i}^{v}\right\rangle .
$$

Now from Theorem 2.4, we know that

$$
\left\langle L_{n, d-2 i}^{v}, L_{n, d-2 i}^{v}\right\rangle=\frac{1}{N(n, d-2 i)} \quad \text { and } \quad L_{n, d-2 i}^{v}=1 .
$$

Thus it follows that $c=1$ as desired.

Remark 4.2. For even $d=2 k$ we may rewrite $p_{v}$ as

$$
p_{v}=\sum_{i=0}^{2 k} N(n, 2 i) r^{2 k-2 i} L_{n, 2 i}^{v}
$$

Corollary 4.3. Let $f \in P_{n, d}$ be such that

$$
\frac{\|f\|_{\infty}}{\|f\|} \geq \frac{\|g\|_{\infty}}{\|g\|} \quad \text { for all nonzero } \quad g \in P_{n, d} .
$$

Then $f$ is a scalar multiple of $p_{e_{n}}$, up to a rotation of $\mathbb{R}^{n}$, and

$$
\frac{\|f\|_{\infty}}{\|f\|}=\sqrt{D(n, d)}
$$

Proof. By applying an appropriate rotation and rescaling we may assume that

$$
\|f\|_{\infty}=f\left(e_{n}\right)=1
$$

We observe that $f$ lies in the affine hyperplane $T$ of all polynomials with value 1 at $e_{n}$ and furthermore $f$ is the shortest form on this hyperplane by the assumption that

$$
\frac{\|f\|_{\infty}}{\|f\|} \geq \frac{\|g\|_{\infty}}{\|g\|} \quad \text { for all } \quad g \in P_{n, d}
$$

Thus $f$ is perpendicular to $T$ and from Theorem 4.1 it follows that $f$ is a multiple of $p_{e_{n}}$.

Let $C^{*}$ denote the dual cone of $C$,

$$
C^{*}=\left\{f \in P_{n, 2 k} \mid\langle f, g\rangle \geq 0 \text { for all } g \in C\right\}
$$


Corollary 4.4. $\quad C^{*}$ is the conical hull of the orbit of $p_{e_{n}}$, where

$$
p_{e_{n}}=\sum_{i=0}^{2 k} N(n, 2 i) r^{2 k-2 i} L_{n, 2 i}
$$

Proof. Let $K$ be the conical hull of the points $p_{v}$ for all $v \in S^{n-1}$,

$$
K=\left\{\sum_{i} \lambda_{i} p_{v_{i}} \mid v_{i} \in S^{n-1} \text { and } \lambda_{i} \in \mathbb{R}, \lambda_{i} \geq 0\right\} .
$$

Consider $K^{*}$,

$$
\begin{aligned}
K^{*} & =\left\{f \in P_{n, 2 k} \mid\langle f, g\rangle \geq 0 \text { for all } g \in K\right\} \\
& =\left\{f \in P_{n, 2 k} \mid\left\langle f, p_{v}\right\rangle \geq 0 \text { for all } v \in S^{n-1}\right\} .
\end{aligned}
$$

From Theorem 4.1, we know that $\left\langle f, p_{v}\right\rangle=f(v)$, and therefore $K^{*}=C$. Since $C$ is a closed cone, by the BiPolar Theorem it follows that $K=C^{*}$.

Now let $A \in S O(n)$ be such that $A w=v$. Then we note that $A L_{n, 2 i}^{v}=L_{n, 2 i}^{w}$ and therefore

$$
A p_{v}=p_{w} .
$$

Thus the set of $p_{v}$ for all $v \in S^{n-1}$ is the same as the orbit of $p_{e_{n}}$ and we obtain the desired result.

\section{John's Ellipsoid of the Cone of Nonnegative Polynomials}

In this section we compute John's ellipsoid of $\bar{C}_{n, 2 k}$. Recall that $M$ is the hyperplane of all forms of integral 1 on $S^{n-1}$. If we regard the point $r^{2 k}=\left(x_{1}^{2}+\cdots+x_{n}^{2}\right)^{k}$ as the origin in $M$, then the inner product $\langle$,$\rangle induces an inner product in M$ which we denote $\langle,\rangle_{M}$,

$$
\langle f, g\rangle_{M}=\left\langle f-r^{2 k}, g-r^{2 k}\right\rangle \quad \text { for } \quad f, g \in M .
$$

Recall that $C^{*}$ is the dual cone of $C$, and define $\bar{C}^{*}$ by intersecting $C^{*}$ with the hyperplane $M$,

$$
\bar{C}^{*}=C^{*} \cap M .
$$

We now establish a relationship between $\bar{C}$ and $\bar{C}^{*}$ in terms of $\langle,\rangle_{M}$.

Lemma 5.1. Let $\bar{C}^{\circ}$ be the polar of $\bar{C}$ with respect to $\langle,\rangle_{M}$. Then

$$
\bar{C}^{\circ}=-\bar{C}^{*}+2 r^{2 k} \text {. }
$$

Proof. We observe that

$$
\begin{aligned}
\bar{C}^{\circ} & =\left\{f \in M \mid\langle f, g\rangle_{M} \leq 1 \text { for all } g \in \bar{C}\right\} \\
& =\left\{f \in M \mid\left\langle f-r^{2 k}, g-r^{2 k}\right\rangle \leq 1 \text { for all } g \in \bar{C}\right\} .
\end{aligned}
$$


Since both $f$ and $g$ have integral 1 on $S^{n-1}$, it follows that

$$
\left\langle f, r^{2 k}\right\rangle=\left\langle g, r^{2 k}\right\rangle=1 \text {, }
$$

and therefore

$$
\bar{C}^{\circ}=\{f \in M \mid\langle f, g\rangle \leq 2 \text { for all } g \in \bar{C}\} .
$$

Thus

$$
-\bar{C}^{\circ}+2 r^{2 k}=\{f \in M \mid\langle f, g\rangle \geq 0 \text { for all } g \in \bar{C}\}=\bar{C}^{*} \text {. }
$$

Theorem 5.2. The Loewner ellipsoid $E$ of $\bar{C}^{*}$ is a ball with center $r^{2 k}$ and radius

$$
\sqrt{D(n, 2 k)-1}=\sqrt{\left(\begin{array}{c}
n+2 k-1 \\
2 k
\end{array}\right)-1} .
$$

Proof. From Corollary 4.4 it follows that $\bar{C}^{*}$ is the convex hull of the orbit of $p_{e_{n}}$. We also know from Theorem 2.1 that the representation of $S O(n)$ is multiplicity free. Therefore we can apply Theorem 3.1. The irreducible subspaces are $H_{n, 2 i}^{*}$ for $1 \leq i \leq k$. Let $l_{2 i}(f)$ denote the projection of $f$ into $H_{n, 2 i}^{*}$ and then

$$
\left\|l_{2 i}\left(p_{e_{n}}\right)\right\|^{2}=\left\|N(n, 2 i) L_{n, 2 i}\right\|^{2}=N(n, 2 i) .
$$

The result now follows from Theorem 3.1.

Theorem 5.3. John's ellipsoid $D$ of $\bar{C}$ is a ball with center $r^{2 k}$ and radius

$$
\frac{1}{\sqrt{D(n, 2 k)-1}}=\frac{1}{\sqrt{\left(\begin{array}{c}
n+2 k-1 \\
2 k
\end{array}\right)-1}} .
$$

Proof. From Lemma 5.1 we know that

$$
\bar{C}^{\circ}=-\bar{C}^{*}+2 r^{2 k} \text {. }
$$

Therefore the Loewner ellipsoid of $\bar{C}^{\circ}$ is a ball with center $r^{2 k}$ and radius $\sqrt{D(n, 2 k)-1}$. By Proposition 2.6 we know that John's ellipsoid of $\bar{C}$ is the polar of the Loewner ellipsoid of $\bar{C} \circ$ and the theorem follows.

\section{Coefficient of Symmetry of the Cone of Nonnegative Polynomials}

In this section we compute the coefficient of symmetry of $\bar{C}$ with respect to $r^{2 k}$. We begin by showing that all forms symmetric with respect to an axis are sums of squares of forms.

Definition. For $0 \leq a \leq 1$, let

$$
q_{a}(x)=x_{n}^{2}-a r^{2}=(1-a) x_{n}^{2}-a\left(x_{1}^{2}+\cdots+x_{n-1}^{2}\right) .
$$


Definition. For $f \in P_{n, d}$ let $V(f)$ be the vanishing set of $f$,

$$
V(f)=\left\{x \in \mathbb{R}^{n} \mid f(x)=0\right\} .
$$

Lemma 6.1. Let $f(x) \in P_{n, 2 k}$ be a nonnegative form and suppose that $f$ is fixed by $J(n, v)$ for some $v \in \mathbb{R}^{n}$. Then $f$ is a sum of squares of forms.

Proof. We induct on $k$.

Base case: $k=1 . \quad$ In this case we are dealing with homogeneous quadratics and all nonnegative homogeneous quadratics are sums of squares.

Inductive step: $k \Rightarrow k+1$. Applying a suitable rotation of $\mathbb{R}^{n}$, we may assume that $f$ is fixed by $J\left(n, e_{n}\right)$. It will suffice to show the lemma for $f$ with a zero, since we can consider the form

$$
f-\alpha r^{2 k}
$$

where $\alpha$ is the minimum of $f$ on $S^{n-1}$. Since $f$ has a zero, and $f$ is fixed by $J\left(n, e_{n}\right)$ it follows that $V(f)$ is a nonempty subset of $\mathbb{R}^{n}$ that is fixed by $J\left(n, e_{n}\right)$. Hence $V(f)$ contains $V_{q_{a}}$ for some $a \in[0,1]$.

We first deal with the two degenerate cases:

If $a=1$, then

$$
q_{1}=-\left(x_{1}^{2}+\cdots+x_{n-1}^{2}\right) \quad \text { and } \quad V_{q_{a}}=\left\{\lambda e_{n} \mid \lambda \in \mathbb{R}\right\} .
$$

Since $f\left(e_{n}\right)=0$ we can write

$$
f=\sum_{i=0}^{2 k-1} x_{n}^{i} g_{i}
$$

where $g_{i}$ depend only on $x_{1}, \ldots, x_{n-1}$. Since $f$ is fixed by $J\left(n, e_{n}\right)$, it follows that $g_{i}$ is fixed by $J\left(n, e_{n}\right)$ for all $0 \leq i \leq 2 k-1$. Since $g_{i}$ depends only on $x_{1}, \ldots, x_{n-1}$, we see that $g_{i}$ is fixed by $S O(n-1)$. Then $i$ must be even and

$$
g_{2 i}=\lambda_{i}\left(x_{1}^{2}+\cdots+x_{n-1}^{2}\right)^{k-i} \quad \text { for some } \quad \lambda_{i} \in \mathbb{R} .
$$

Thus $x_{1}^{2}+\cdots+x_{n-1}^{2}$ divides $f$. We write $f=\left(x_{1}^{2}+\cdots+x_{n-1}^{2}\right) g$ and $g$ is the sum of squares by induction, and then so is $f$.

If $a=0$, then $q_{0}(x)=x_{n}^{2}$ and $x_{n}$ divides $f$, but since $f$ is nonnegative, it follows that $x_{n}^{2}$ divides $f$ and $f=x_{n}^{2} g$. By induction, $g$ is a sum of squares, and then $f$ is as well.

For $0<a<1$, let $I=I\left(V_{q_{a}}\right)$ be the vanishing ideal of $V_{q_{a}}$ :

$$
I=\left\{f \in \mathbb{R}\left[x_{1}, \ldots, x_{n}\right] \mid f(x)=0 \text { for all } x \in V_{q_{a}}\right\},
$$

where $\mathbb{R}\left[x_{1}, \ldots, x_{n}\right]$ is the ring of real polynomials in $n$ variables. We will show that $I$ is a principal ideal generated by $q_{a}$.

Let $g \in I$. By reducing modulo $q_{a}$ we may write

$$
g=b q_{a}+x_{n} c+d,
$$


where $c$ and $d$ are polynomials that depend only on $x_{1}, \ldots, x_{n-1}$. Let

$$
h=g-b p_{a}=x_{n} c+d .
$$

We observe that $h \in I$ and also $h\left(x_{1}, \ldots, x_{n-1},-x_{n}\right) \in I$, since $V\left(q_{a}\right)$ is fixed by reflection about the $e_{1}, \ldots, e_{n-1}$ hyperplane. Thus $-x_{n} c+d \in I$, and then $x_{n} c$ and $d$ are in $I$. However, since $a>0$, the vanishing set of $q_{a}$ intersects the hyperplane $x_{n}=0$ only at the origin. Thus we see that $c \in I$. Also, $c$ and $d$ only depend on the first $n-1$ variables. Therefore, since $a<1$, we see that $c$ and $d$ vanish on the entire hyperplane spanned by $e_{1}, \ldots, e_{n-1}$. Hence,

$$
c=d \equiv 0 .
$$

Thus $I=\left(q_{a}\right)$.

Since $I$ is a principal ideal generated by $q_{a}$ and $f \in I$ it follows that $q_{a}$ divides $f$, and we can write $f=q_{a} g$. Now we note that $q_{a}(x) \geq 0$ for $x \in S^{n-1}$ with $x_{n}^{2} \geq$ $a$, and $q_{a}(x)<0$ for $x \in S^{n-1}$ with $x_{n}^{2}<a$. Since $q_{a} g \geq 0$, it follows that

$$
g(x)=0 \quad \text { for all } \quad x \in V\left(p_{a}\right),
$$

otherwise the sign of $g$ does not change in the neighborhood of some $x \in V\left(q_{a}\right)$, which yields a contradiction since $a<1$. Thus $g \in I$ and therefore $q_{a}$ divides $g$. Hence $q_{a}^{2}$ divides $f$. We write $f=q_{a}^{2} h$ and $h$ is a sum of squares by induction.

Remark 6.2. From the proof of Lemma 6.1 it follows that if

$$
V\left(q_{a}\right) \subseteq V(f) \quad \text { with } \quad 0 \leq a<1
$$

for some nonnegative $f \in P_{n, 2 k}$, not necessarily symmetric with respect to $J\left(n, e_{n}\right)$, then

$$
q_{a}^{2} \text { divides } f \text { for } 0<a<1 \text { and } x_{n}^{2} \text { divides } f \text { if } a=0 .
$$

Our goal is to compute the coefficient of symmetry of $\bar{C}$. We begin with the crucial integral inequality.

Definition. Let Max denote the maximal $L^{\infty}$ norm for the functions in $\bar{C}$,

$$
\operatorname{Max}=\max _{f \in \bar{C}}\|f\|_{\infty} .
$$

Theorem 6.3. Let $f \in \bar{C}$ be such that $\|f\|_{\infty}=$ Max. Then

$$
f=\frac{1}{D(n, k)}\left(\sum_{l=0}^{\lfloor k / 2\rfloor} N(n, k-2 l) r^{2 l} L_{n, k-2 l}\right)^{2},
$$

up to a rotation of $\mathbb{R}^{n}$, and

$$
\operatorname{Max}=\|f\|_{\infty}=D(n, k) .
$$


Proof. Let $f \in \bar{C}$ be such that $\|f\|_{\infty}=$ Max. Applying a rotation of $\mathbb{R}^{n}$, if necessary, we may assume that $f\left(e_{n}\right)=$ Max. Now let $p$ be the average of $f$ over $J\left(n, e_{n}\right)$,

$$
p=\int_{A \in J\left(n, e_{n}\right)} A f d \mu,
$$

where $\mu$ is the normalized Haar measure on $J\left(n, e_{n}\right)$. Clearly, $p$ is a nonnegative form and

$$
\int_{S^{n-1}} p d \sigma=1 .
$$

Thus $p \in \bar{C}$. Also, $\|p\|_{\infty}=\|f\|_{\infty}=\operatorname{Max}$, since $p\left(e_{n}\right)=f\left(e_{n}\right)$. Since $p$ is the average of $f$ over $J\left(n, e_{n}\right)$, it follows that $p$ is fixed by $J\left(n, e_{n}\right)$. Then from Lemma 6.1 we see that $p$ is a sum of squares.

Since $p \in \overline{S q}$, it is a convex combination of extreme points of $\overline{S q}$, and an extreme point of $\overline{S q}$ must be a square. Thus we see that

$$
p=\sum \lambda_{i} h_{i}^{2} \quad \text { with } \quad \lambda_{i}>0, \quad \sum \lambda_{i}=1,
$$

where $h_{i} \in P_{n, k}$. Therefore,

$$
\operatorname{Max}=p\left(e_{n}\right)=\sum \lambda_{i} h_{i}^{2}\left(e_{n}\right) .
$$

However,

$$
\operatorname{Max} \geq\left\|h_{i}^{2}\right\|_{\infty} \geq h_{i}^{2}\left(e_{n}\right) \quad \text { and therefore } \quad\left\|h_{i}^{2}\right\|_{\infty}=h_{i}^{2}\left(e_{n}\right)=\text { Max. }
$$

Thus there exists $h \in P_{n, k}$ such that $h^{2} \in \overline{S q}$ and

$$
\left\|h^{2}\right\|_{\infty}=\text { Max. }
$$

Then we observe that

$$
\frac{\|h\|_{\infty}}{\|h\|} \geq \frac{\|g\|_{\infty}}{\|g\|} \quad \text { for all } \quad g \in P_{n, k} .
$$

Then from Corollary 4.3 it follows that

$$
h=\frac{1}{\sqrt{D(n, k)}} \sum_{i=0}^{\lfloor k / 2\rfloor} N(n, k-2 i) r^{2 i} L_{n, k-2 i},
$$

up to a rotation of $\mathbb{R}^{n}$. Also from Corollary 4.3 we know that

$$
\operatorname{Max}=\left\|h^{2}\right\|_{\infty}=D(n, k) .
$$

Now we will show that up to a rotation of $\mathbb{R}^{n}$, the only form in $\bar{C}$ with maximal $L^{\infty}$ norm is $h^{2}$. We know that all Legendre harmonics are fixed by $J\left(n, e_{n}\right)$. Therefore it follows that $h^{2}$ is also fixed by $J\left(n, e_{n}\right)$. Now we observe that from the proof of Corollary 4.3 it is clear that

$$
h^{2}\left(e_{n}\right)=h^{2}\left(-e_{n}\right)=\left\|h^{2}\right\|_{\infty}=\operatorname{Max},
$$


and $e_{n},-e_{n}$ are the only points where the maximum occurs. Thus, if $A \in S O(n)$ acts on $h$, then it either fixes $h$, or the maximum of $A h$ occurs not at $\pm e_{n}$. Therefore $h^{2}$ is the only square, and thus the only extreme point of $\overline{S q}$, which takes on the value Max at $e_{n}$. Now going back to (3) we see that $p=h^{2}$, since $p$ is a convex linear combination of extreme points of $\overline{S q}$ with value Max at $e_{n}$. Therefore,

$$
h^{2}=\int_{A \in J\left(n, \varepsilon_{n}\right)} A f d \mu .
$$

Now $h^{2}$ lies in the boundary of $\bar{C}$, and thus it must have a zero. However, $h^{2}$ is also the average of $f$ over $J\left(n, e_{n}\right)$ and we know that $f$ is nonnegative. Therefore we see that $V(f)$ contains $V\left(q^{2}\right)$. Since $q^{2}$ is fixed by $J\left(n, e_{n}\right)$, it follows that $V\left(q_{a}\right) \subseteq V\left(h^{2}\right)$, for some $a \in[0,1]$, and since $h\left(e_{n}\right) \neq 0$ it follows that $0 \leq a<1$. Then it follows from Remark 6.2 that we can factor out a square of a form fixed by $J\left(n, e_{n}\right)$ from $h^{2}$ and $f$. Call it $m^{2}$, and let

$$
\tilde{h}^{2}=\frac{h^{2}}{m^{2}} \quad \text { and } \quad \tilde{f}=\frac{f}{m^{2}} .
$$

Again, $\tilde{h}^{2}$ generates an extreme ray of a cone of sums of squares, now of a lesser degree, otherwise $h^{2}$ would not lie on an extreme ray. Since $m$ is fixed by $J\left(n, e_{n}\right)$, we still have

$$
\tilde{h}^{2}=\int_{A \in J\left(n, \varepsilon_{n}\right)} A \tilde{f} d \mu,
$$

because averaging over $J\left(n, e_{n}\right)$ is the same as taking the average over slices of the sphere with hyperplanes perpendicular to $e_{n}$. Thus, again by proof of Lemma 6.1 we can factor out the same square from both $\tilde{h}^{2}$ and $\tilde{f}$ and we can continue with this process, and in the end $f=h^{2}$.

Corollary 6.4. For all $f \in P_{n, k}$,

$$
\|f\|_{\infty} \leq\left(\begin{array}{c}
n+k l-1 \\
k l
\end{array}\right)^{1 / 2 l}\|f\|_{2 l} .
$$

Proof. We apply Theorem 6.3 to $f^{2 l}$. Since $f^{2 l}$ is nonnegative, and $f^{2 l} \in P_{n, 2 k l}$, from Theorem 6.3 we know that

$$
\left\|f^{2 l}\right\|_{\infty} \leq D(n, k l)\left\|f^{2 l}\right\|_{1} .
$$

Since

$$
\left\|f^{2 l}\right\|_{\infty}=\|f\|_{\infty}^{2 l} \quad \text { and } \quad\left\|f^{2 l}\right\|_{1}=\|f\|_{2 l}^{2 l},
$$

by taking the $2 l$ th root of both sides we obtain the desired inequality.

Theorem 6.5. The coefficient of symmetry of $\bar{C}$ with respect to $r^{2 k}$ is

$$
\frac{1}{D(n, k)-1}=\frac{1}{\left(\begin{array}{c}
n+k-1 \\
k
\end{array}\right)-1}
$$


Proof. Let $f \in \partial \bar{C}$, and denote by $\bar{f}$ the polynomial in $\partial \bar{C}$ that is opposite to $f$ with respect to $r^{2 k}$,

$$
\bar{f}=\alpha\left(r^{2 k}-f\right)+r^{2 k} \quad \text { for some } \quad \alpha \in \mathbb{R} \quad \text { such that } \quad \alpha>0 .
$$

Since $\bar{f} \in \partial \bar{C}$, it is a nonnegative form with a zero. Then it follows that

$$
\alpha=\frac{1}{\max _{x \in S^{n-1}} f(x)-1}=\frac{1}{\|f\|_{\infty}-1} .
$$

Thus

$$
\bar{f}=\frac{1}{\|f\|_{\infty}-1}\left(r^{2 k}-f\right)+r^{2 k}
$$

and, since the minimum of $f$ on $S^{n-1}$ is zero,

$$
\|\bar{f}\|_{\infty}=\alpha+1=\frac{\|f\|_{\infty}}{\|f\|_{\infty}-1} .
$$

Also using (4) we see that

$$
\frac{\left\|f-r^{2 k}\right\|}{\left\|\bar{f}-r^{2 k}\right\|}=\frac{\left\|f-r^{2 k}\right\|}{\left\|\left(1 /\left(\|f\|_{\infty}-1\right)\right)\left(r^{2 k}-f\right)\right\|}=\|f\|_{\infty}-1 .
$$

Therefore it follows that the coefficient of symmetry of $\bar{C}$ with respect to $r^{2 k}$ is $1 /$ (Max 1). From Theorem 6.3, we know that $\operatorname{Max}=D(n, k)$, and the result follows.

Corollary 6.6. Let $M_{f}$ denote the maximum of $f$ on $S^{n-1}$ and let $m_{f}$ denote the minimum of $f$ on $S^{n-1}$. Let $\alpha=1 /\left(\begin{array}{c}n+k-1 \\ k\end{array}\right)$. Then

$$
\alpha M_{f}+(1-\alpha) m_{f} \leq \int_{S^{n-1}} f d \sigma \leq(1-\alpha) M_{f}+\alpha m_{f},
$$

and both inequalities are sharp.

Proof. Consider the set $W$ obtained from $\bar{C}$ by subtracting $r^{2 k}$ from all forms in $\bar{C}$,

$$
W=\bar{C}-r^{2 k} \text {. }
$$

We observe that $W$ is the set of all forms of integral zero with minimum at most -1 on $S^{n-1}$.

From the definition of $W$ it follows that the coefficient of symmetry of $W$ around zero is the same as the coefficient of symmetry of $\bar{C}$ around $r^{2 k}$. Thus the coefficient of symmetry of $W$ around zero is

$$
\frac{1}{\left(\begin{array}{c}
n+k-1 \\
k
\end{array}\right)-1}=\frac{\alpha}{1-\alpha} .
$$

However, since

$$
M_{-f}=-m_{f} \quad \text { and } \quad m_{-f}=-M_{f}
$$


it follows that for all $f \in P_{n, 2 k}$ of integral zero,

$$
\frac{\alpha}{1-\alpha} \leq \frac{-M_{f}}{m_{f}} \leq \frac{1-\alpha}{\alpha}
$$

For $f \in P_{n, 2 k}$ consider

$$
\hat{f}=f-\left(\int_{S^{n-1}} f d \sigma\right) r^{2 k}
$$

We have shown above that

$$
\frac{-\left(M_{f}-\int_{S^{n-1}} f d \sigma\right)}{m_{f}-\int_{S^{n-1}} f d \sigma}=\frac{-M_{\hat{f}}}{m_{\hat{f}}} \leq \frac{1-\alpha}{\alpha} .
$$

Thus

$$
\frac{1}{\alpha} \int_{S^{n-1}} f d \sigma \geq M_{f}+\left(\frac{1}{\alpha}-1\right) m_{f},
$$

and one side of the desired inequality follows. The other half is done in the same way.

Corollary 6.7. $\bar{C}$ is contained in ball of radius

$$
\sqrt{D(n, k)-1}=\sqrt{\left(\begin{array}{c}
n+k-1 \\
k
\end{array}\right)-1}
$$

or, equivalently, for all nonnegative $f \in P_{n, 2 k}$,

$$
\|f\|_{2} \leq \sqrt{\left(\begin{array}{c}
n+k-1 \\
k
\end{array}\right)}\|f\|_{1}
$$

Proof. From Theorem 5.3 we know that John's ellipsoid of $\bar{C}$ is a ball of radius $1 / \sqrt{[b] D(n, 2 k)-1}$ around $r^{2 k}$, and the coefficient of symmetry of $\bar{C}$ with respect to $r^{2 k}$ is $1 /(D(n, k)-1)$. We apply Proposition 2.7 , and it follows that therefore $\bar{C}$ is contained in the ball of radius

$$
\frac{\sqrt{D(n, k)-1} \sqrt{D(n, 2 k)-1}}{\sqrt{D(n, 2 k)-1}}=\sqrt{D(n, k)-1}=\sqrt{\left(\begin{array}{c}
n+k-1 \\
k
\end{array}\right)-1},
$$

centered at $r^{2 k}$, as desired.

\section{Cone of Sums of Powers of Linear Forms}

In order to study the cone $L f$ we need to decompose $x_{n}^{2 k}$ as a sum of Legendre harmonics. We begin by recalling the Rodrigues Rule. 
Lemma 7.1. Rodrigues Rule [7]: Let $Q_{n, d}(t)$ be the Legendre polynomial defined in Section 2. Then

$$
\int_{-1}^{+1} f(t) Q_{n, d}(t)\left(1-t^{2}\right)^{(n-3) / 2} d t=R_{d}(n) \int_{-1}^{+1} f^{(n)}(t)\left(1-t^{2}\right)^{(2 d+n-3) / 2},
$$

where $R_{d}(n)$ is the Rodrigues constant:

$$
R_{d}(n)=\frac{\Gamma((n-1) / 2)}{2^{n} \Gamma((4 k+n-1) / 2)} .
$$

Since $x_{n}^{2 k}$ is symmetric and is fixed by the action of $J\left(n, e_{n}\right)$ it decomposes as a sum of the Legendre harmonics. The next theorem gives the precise decomposition.

\section{Theorem 7.2.}

$$
\frac{x_{n}^{2 k}}{\int_{S^{n-1}} x_{n}^{2 k} d \sigma}=\sum_{l=0}^{k} \frac{k ! \Gamma((2 k+n) / 2)}{(k-l) ! \Gamma((2 k+2 l+n) / 2)} N(n, 2 l) r^{2 k-2 l} L_{n, 2 l} .
$$

Proof. We first recall the well-known fact that

$$
\int_{S^{n-1}} x_{n}^{2 k} d \sigma=\frac{\Gamma((2 k+1) / 2) \Gamma(n / 2)}{\sqrt{\pi} \Gamma((n+2 k) / 2)} .
$$

See, for example, [2].

Since $x_{n}^{2 k}$ is fixed by $J\left(n, e_{n}\right)$, we know that it decomposes as a sum of Legendre harmonics of even degrees. Therefore it suffices to compute

$$
\left\langle x_{n}^{2 k}, r^{2 l} L_{n, 2 k-2 l}\right\rangle=\int_{S^{n-1}} x_{n}^{2 k} L_{n, 2 k-2 l} d \sigma .
$$

On $S^{n-1}$ both $x_{n}^{2 k}$ and $L_{n, 2 k-2 l}$ are functions of the last coordinate, and hence this integral translates into

$$
\frac{\left|S^{n-2}\right|}{\left|S^{n-1}\right|} \int_{-1}^{+1} t^{2 k} Q_{n, 2 k-2 l}(t)\left(1-t^{2}\right)^{(n-3) / 2} d t,
$$

where $\left|S^{n-1}\right|$ denotes the surface area of $S^{n-1}$. Now we apply the Rodrigues Rule to

$$
\int_{-1}^{+1} t^{2 k} Q_{n, 2 k-2 l}(t)\left(1-t^{2}\right)^{(n-3) / 2} d t
$$

and get

$$
\frac{(2 k) !}{(2 l) !} R_{2 k-2 l}(n) \int_{-1}^{+1} t^{2 l}\left(1-t^{2}\right)^{(4 k-4 l+n-3) / 2} d t .
$$

This we can interpret back as an integral over the sphere of dimension $4 k-4 l+n-1$ and we obtain

$$
\frac{(2 k) !\left|S^{4 k-4 l+n-1}\right|}{(2 l) !\left|S^{4 k-4 l+n-2}\right|} R_{2 k-2 l}(n) \int_{S^{4 k-4 l+n-1}} x_{n}^{2 l} d \sigma
$$


Next we substitute in (5) to get

$$
\frac{(2 k) !\left|S^{4 k-4 l+n-1}\right| \Gamma((2 l+1) / 2) \Gamma((4 k-4 l+n) / 2)}{\sqrt{\pi}(2 l) !\left|S^{4 k-4 l+n-2}\right| \Gamma((4 k-2 l+n) / 2)} R_{2 k-2 l}(n) .
$$

Now,

$$
\left|S^{n-1}\right|=\frac{\pi^{n / 2}}{\Gamma(n / 2)},
$$

and thus we get

$$
\frac{(2 k) ! \Gamma((2 l+1) / 2) \Gamma((4 k-4 l+n-1) / 2)}{(2 l) ! \Gamma((4 k-2 l+n) / 2)} R_{2 k-2 l}(n) .
$$

Substituting in the value of $R_{2 k-2 l}(n)$ we obtain

$$
\int_{-1}^{+1} t^{2 k} Q_{n, 2 k-2 l}(t)\left(1-t^{2}\right)^{(n-3) / 2} d t=\frac{(2 k) ! \Gamma((2 l+1) / 2) \Gamma((n-1) / 2)}{2^{2 k-2 l}(2 l) ! \Gamma((4 k-2 l+n) / 2)} .
$$

Thus we get that

$$
\begin{aligned}
\left\langle x_{n}^{2 k}, r^{2 l} L_{n, 2 k-2 l}\right\rangle & =\frac{\left|S^{n-2}\right|(2 k) ! \Gamma((2 l+1) / 2) \Gamma((n-1) / 2)}{\left|S^{n-1}\right| 2^{2 k-2 l}(2 l) ! \Gamma((4 k-2 l+n) / 2)} \\
& =\frac{(2 k) ! \Gamma((2 l+1) / 2) \Gamma(n / 2)}{\sqrt{\pi} 2^{2 k-2 l}(2 l) ! \Gamma((4 k-2 l+n) / 2)} .
\end{aligned}
$$

Now the doubling rule for the gamma function says that

$$
2^{x-1} \Gamma\left(\frac{x}{2}\right) \Gamma\left(\frac{x+1}{2}\right)=\sqrt{\pi} \Gamma(x) .
$$

Applying this to $x=2 l+1$ we get

$$
\Gamma\left(\frac{2 l+1}{2}\right)=\frac{\sqrt{\pi} \Gamma(2 l+1)}{2^{2 l} \Gamma(l+1)} .
$$

Substituting (7) into (6) we have

$$
\left\langle x_{n}^{2 k}, r^{2 l} L_{n, 2 k-2 l}\right\rangle=\frac{(2 k) ! \Gamma(n / 2)}{l ! 2^{2 k} \Gamma((4 k-2 l+n) / 2)} .
$$

Thus using (5),

$$
t \frac{\left\langle x_{n}^{2 k}, r^{2 l} L_{n, 2 k-2 l}\right\rangle}{\int_{S^{n-1}} x_{n}^{2 k} d \sigma}=\frac{\sqrt{\pi}(2 k) ! \Gamma((2 k+n) / 2)}{2^{2 k} l ! \Gamma((4 k-2 l+n) / 2) \Gamma((2 k+1) / 2)} .
$$

Now we again apply the doubling rule this time to $x=2 k+1$ to get

$$
\Gamma\left(\frac{2 k+1}{2}\right)=\frac{\sqrt{\pi} \Gamma(2 k+1)}{2^{2 k} \Gamma(k+1)},
$$


which we substitute into (8):

$$
\frac{\left\langle x_{n}^{2 k}, r^{2 l} L_{n, 2 k-2 l}\right\rangle}{\int_{S^{n-1}} x_{n}^{2 k} d \sigma}=\frac{k ! \Gamma((2 k+n) / 2)}{l ! \Gamma((4 k-2 l+n) / 2)} .
$$

Now recall that

$$
\left\|r^{2 l} L_{n, 2 k-2 l}\right\|^{2}=\left\|L_{n, 2 k-2 l}\right\|^{2}=\frac{1}{N(n, 2 k-2 l)}
$$

and the desired result follows.

We now make a crucial definition. We define a family of linear operators $T_{2 m, 2 k}$ that averages a form $p$ in $P_{n, 2 k}$ over the $2 m$ th powers of linear forms $\langle x, v\rangle$ for $v$ in $S^{n-1}$ and $m \geq k$.

Definition. Let $T_{2 m, 2 k}: P_{n, 2 k} \rightarrow P_{n, 2 k}$ be a linear operator defined by

$$
\left(T_{2 m, 2 k} p\right)(x)=\frac{\int_{S^{n-1}} p(v)\langle x, v\rangle^{2 m} d \sigma(v)}{\int_{S^{n-1}} x_{n}^{2 m} d \sigma} \quad \text { for } \quad x, v \in S^{n-1} \text {. }
$$

The fact that the result of the averaging is a polynomial of degree $2 k$ rather than $2 m$ follows from the following observation. Since we only look at the unit sphere we can replace $p$ with $p r^{2 m-2 k}$ and the resulting polynomial will be of degree $2 m$ but it will be divisible by $r^{2 m-2 k}$, and thus on $S^{n-1}$ it will be the same as a polynomial of degree $2 k$.

Remark 7.3. It will follow from Lemma 7.4 that the operators $T_{2 m, 2 k}$ have been defined in a different form by Reznick in [9].

We observe that $T_{2 m, 2 k}$ maps nonnegative forms to the sums of powers of linear forms. The following lemma shows the precise action of $T_{2 m, 2 k}$ on $P_{n, 2 k}$.

\section{Lemma 7.4.}

$$
T_{2 m, 2 k}(f)=\sum_{i=0}^{k} \frac{m ! \Gamma((2 m+n) / 2)}{(m-i) ! \Gamma((2 m+2 i+n) / 2)} l_{2 i}(f) .
$$

Proof. We rewrite $T_{2 m, 2 k} f$ as

$$
\left(T_{2 m, 2 k} f\right)(x)=\left\langle f \frac{\langle x, v\rangle^{2 m}}{\int_{S^{n-1}}\langle x, v\rangle^{2 m} d \sigma(v)}\right\rangle .
$$

We apply Theorem 7.2 and it follows that

$$
\left(T_{2 m, 2 k} f\right)(x)=\left\langle f \sum_{i=0}^{m} \frac{m ! \Gamma((2 m+n) / 2)}{(m-i) ! \Gamma((2 m+2 i+n) / 2)} N(n, 2 i) L_{n, 2 i}^{v}\right\rangle
$$


Now we decompose $f$ as a sum of spherical harmonics and observe that

$$
\left(T_{2 m, 2 k} f\right)(x)=\sum_{i=0}^{k}\left\langle l_{2 i}(f) \frac{m ! \Gamma((2 m+n) / 2)}{(m-i) ! \Gamma((2 m+2 i+n) / 2)} N(n, 2 i) L_{n, 2 i}^{v}\right\rangle .
$$

We recall that by Theorem 4.1

$$
\left\langle l_{2 i}(f), N_{n, 2 i} L_{n, 2 i}^{v}\right\rangle=\left(l_{2 i} f\right)(v) .
$$

Therefore

$$
T_{2 m, 2 k}(f)=\sum_{i=0}^{k} \frac{m ! \Gamma((2 m+n) / 2)}{(m-i) ! \Gamma((2 m+2 i+n) / 2)} l_{2 i}(f) .
$$

Remark 7.5. It follows from Lemma 7.4 that $T_{2 m, 2 k}$ is a diagonal operator on the harmonic subspaces of $P_{n, 2 k}$. Thus $T_{2 m, 2 k}$ commutes with the action of $S O(n)$.

Theorem 7.6. The Loewner ellipsoid of $\overline{L f}$ is given by the inequality

$$
\sum_{i=1}^{k}\left(\frac{(k-i) ! \Gamma((2 k+2 i+n) / 2)}{k ! \Gamma((2 k+n) / 2)}\right)^{2}\left\|l_{2 i}(f)\right\|^{2} \leq D_{n, 2 k}-1,
$$

and the coefficient of symmetry of $\overline{L f}$ is

$$
\frac{1}{D(n, k)-1}=\frac{1}{\left(\begin{array}{c}
n+k-1 \\
k
\end{array}\right)-1} .
$$

Proof. By Lemma 7.4 and Theorem 7.2,

$$
T_{2 k, 2 k}\left(p_{e_{n}}\right)=\sum_{i=0}^{k} \frac{k ! \Gamma((2 k+n) / 2)}{(k-i) ! \Gamma((2 k+2 i+n) / 2)} N(n, 2 i) L_{n, 2 i}=\frac{x_{n}^{2 k}}{\int_{S^{n-1}} x_{n}^{2 k} d \sigma} .
$$

Since $T_{2 k, 2 k}$ commutes with the action of $S O(n)$, it follows that

$$
T_{2 k, 2 k}\left(\bar{C}^{*}\right)=\overline{L f} \text {. }
$$

Therefore $T_{2 k, 2 k}$ maps the Loewner ellipsoid of $\bar{C}^{*}$ to the Loewner ellipsoid of $\overline{L f}$. By Theorem 5.2 the Loewner ellipsoid of $\overline{L f}$ is a ball with center $r^{2 k}$ and of radius $\sqrt{D(n, k)-1}$. The inequality for the Loewner ellipsoid of $\overline{L f}$ follows.

By Lemma 5.1 we know that $\bar{C}^{*}$ and $\bar{C}$ are after a reflection polar to each other. Therefore they have the same coefficient of symmetry with respect to $r^{2 k}$. Since $T_{2 k, 2 k}$ fixes $r^{2 k}$, it follows that the coefficient of symmetry of $\overline{L f}$ is the same as the coefficient of symmetry of $\bar{C}^{*}$, which by Theorem 6.5 is $(D(n, k)-1)^{-1}$.

Corollary 7.7. $\overline{L f}$ contains a ball of radius

$$
\frac{k ! \Gamma((2 k+n) / 2)}{\Gamma((4 k+n) / 2) \sqrt{D(n, k)-1}}
$$

centered at $r^{2 k}$. 
Proof. The coefficient

$$
\frac{(k-i) ! \Gamma((2 k+2 i+n) / 2)}{k ! \Gamma((2 k+n) / 2)} \quad \text { for } \quad 0 \leq i \leq k
$$

is clearly maximized when $i=k$. Thus the Loewner ellipsoid of $\overline{L f}$ contains a ball of radius

$$
\frac{k ! \Gamma((2 k+n) / 2) \sqrt{D(n, 2 k)-1}}{\Gamma((4 k+n) / 2)} .
$$

From Proposition 2.7 and Theorem 7.6 we know that $\overline{L f}$ will contain its Loewner ellipsoid shrunk by the factor of

$$
\frac{1}{\sqrt{(D(n, 2 k)-1)(D(n, k)-1)}} .
$$

Now the corollary follows.

Definition. Let $K(2 m)$ be the cone in $P_{n, 2 k}$ of forms whose restrictions to the sphere are linear combinations of $2 m$ th powers of linear forms on $S^{n-1}$. Equivalently $K(2 m)$ is the cone of forms in $P_{n, 2 k}$ that multiplied by $r^{2 m-2 k}$ become sums of powers of linear forms

$$
K(2 m)=\left\{f \in P_{n, 2 k} \mid\left(x_{1}^{2}+\cdots+x_{n}^{2}\right)^{m-k} f \in L f_{n, 2 m}\right\} .
$$

We define $\bar{K}(2 m)$ by intersecting $K$ with the hyperplane of forms of integral 1 on $S^{n-1}$.

Theorem 7.8. $\quad$ Let $\alpha=\operatorname{dim} P_{n, 2 k}-1$. Then

$$
\left(\frac{\operatorname{vol} \bar{K}(2 m)}{\operatorname{vol} \bar{C}}\right)^{1 / \alpha} \geq \frac{m ! \Gamma((2 m+n) / 2)}{(m-k) ! \Gamma((2 m+2 k+n) / 2)} .
$$

Proof. We observe that from the definition of $T_{2 m, 2 k}$ it follows that $T_{2 m, 2 k}$ maps $C$ into $K(2 m)$. Since $T_{2 m, 2 k}$ fixes $r^{2 k}$, it follows that $T_{2 m, 2 k}$ maps $\bar{C}$ into $\bar{K}(2 m)$. However, from Lemma $7.4 T_{2 m, 2 k}$ acts on $H_{n, 2 i}^{*}$ by shrinking it by a factor of

$$
\frac{m ! \Gamma((2 m+n) / 2)}{(m-i) ! \Gamma((2 m+2 i+n) / 2)} \text {. }
$$

This coefficient is clearly minimized when $i=k$ and then the theorem follows.

Corollary 7.9. Let $\varepsilon \geq 0$ and let $m=\left(2 k^{2}+k n\right) / \varepsilon$. Then

$$
\left(\frac{\operatorname{vol} \bar{K}(2 m)}{\operatorname{vol} \bar{C}}\right)^{1 / \alpha} \geq 1-\varepsilon
$$


Remark 7.10. The volume ratio

$$
\left(\frac{\operatorname{vol} \bar{K}(2 m)}{\operatorname{vol} \bar{C}}\right)^{1 / \alpha}
$$

approaches 1 as $m$ tends to infinity. Therefore every strictly positive form lies in some $K(2 m)$ (see [9])

\section{Acknowledgements}

The author thanks Alexander Barvinok for many discussions and support in writing this paper.

\section{References}

1. K. Ball, An elementary introduction to modern convex geometry, in Flavors of Geometry, pp. 1-58, Mathematical Science Research Institute Publication 31, Cambridge University Press, Cambridge, 1997.

2. A.I. Barvinok, Estimating $L^{\infty}$ norms by $L^{2 k}$ norms for functions on orbits, Foundations of Computational Mathematics, to appear.

3. J. Bochnak, M. Coste and M-F. Roy, Real Algebraic Geometry, Springer-Verlag, Berlin, 1998.

4. J. Duoandikoetxea, Reverse Hölder inequalities for spherical harmonics, Proc. Amer. Math. Soc. 101(3) (1987), 487-491.

5. D. Hilbert, Über die Darstellung definiter Formen als Summe von Formenquadraten, Math. Ann. 32(1888), 342-350 Ges Abh. vol. 2, pp. 415-436, Chelsea, New York, 1965.

6. F. John, Extremum problems with inequalities as subsidiary conditions, Studies and Essays Presented to R. Courant on his 60th Birthday, pp. 187-204, Interscience, New York, 1948.

7. C. Müller, Analysis of Spherical Symmetries in Euclidean Spaces, Springer-Verlag, New York, 1998.

8. B. Reznick, Sums of even powers of real linear forms, Mem. Amer. Math. Soc. 96 (1992), no. 463.

9. B. Reznick, Some concrete aspects of Hilbert's 17th Problem, Contemp. Math., 253 (2000), 251-272.

10. B. Simon, Representations of Finite and Compact Groups, Graduate Studies in Mathematics 10, American Mathematical Society, Providence, RI, 2001.

11. C. Sogge, Oscillatory integrals and spherical harmonics, Duke Math. J. 53(1) (1986), 43-65.

12. G. Szegö, Orthogonal Polynomials, American Mathematical Society Colloquium Publications 23, American Mathematical Society, Providence, RI, 1939.

13. N. Ja. Vilenkin, Special Functions and the Theory of Group Representations, Translations of Mathematical Monographs 22, American Mathematical Society, Providence, RI, 1968.

Received October 27, 2002, and in final form November 6, 2003. Online publication June 4, 2004. 